\title{
PROTOCOLO DE ANÁLISE RÁPIDA: ALTERNATIVA PARA AVALIAR QUALIDADE AMBIENTAL EM RIACHOS DE CABECEIRA EM MATA ATLÂNTICA, SUL DO BRASIL
}

\author{
Rapid analysis protocol: alternative to assess environmental quality in \\ stream headwaters in Atlantic Forest, southern Brazil
}

\author{
Jair Marques da Silva ${ }^{1}$; Caciane Larissa Rauch ${ }^{2}$; Cristiane Biasi ${ }^{1}$; Luiz Ubiratan Hepp ${ }^{1}$; \\ Vanderlei Secretti Decian ${ }^{1}$; Rozane Maria Restello
}

\begin{abstract}
${ }^{1}$ PPG Ecologia, Departamento de Ciências Biológicas. URI Erechim. E-mail: rrozane@uricer.edu.br
${ }^{2}$ Curso de Ciências Biológicas - Bacharelado. URI Erechim.
\end{abstract}

Data do recebimento: 03/09/2019 - Data do aceite: 06/10/2019

RESUMO: Este trabalho teve por objetivo elaborar um Protocolo de Análise Rápida (PAR) destinado à avaliação da diversidade de habitats e do nível de conservação de riachos, como ferramenta para o monitoramento e a gestão de bacias hidrográficas. Para a elaboração do PAR foram selecionadas duas Bacias Hidrográficas, uma no Rio Grande do Sul e uma em Santa Catarina. A partir dos dados de uso e ocupação da terra, foram selecionados quatro trechos de riachos onde foram elencados 19 parâmetros. Para cada característica do parâmetro atribui-se uma pontuação de 0 a 4 pontos, de acordo com uma condição de estresse ambiental. Ao final, a somatória da pontuação dos parâmetros classifica os trechos em natural, alterado e impactado. Dos trechos selecionados, apenas um em cada bacia apresentou-se natural; os demais, alterados ou impactados. O protocolo elaborado foi validado pela aplicação do índice Biological Monitoring Working Party (BMWP) que usa macroinvertebrados bentônicos como bioindicadores. Esta validação demonstrou resultados semelhantes em 50\% dos trechos avaliados, com uma correlação positiva entre os resultados do PAR e do BMWP $(\mathrm{r}=0,85)$. Assim, o uso de PAR pode ser considerado uma ferramenta de avaliação eficiente, além de proporcionar a gestão participativa e integrada que é preconizada pela Política Nacional de Recursos Hídricos.

Palavras-chave: Avaliação de Habitats. Recursos Hídricos. Gestão Ambiental. 
ABSTRACT: The objective of this work was to elaborate a Rapid Assessment Protocol (RAP), intended to assess the diversity of habitats and stream conservation level as a tool for watershed monitoring and management. Two river basins were selected for the RAP elaboration, one in Rio Grande do Sul and one in Santa Catarina. From the land use and occupation data, four stretches of the streams were selected in which 19 parameters were listed. A score of 0 to 4 points is assigned to each characteristic of the parameter, according to an environmental stress condition. At the end, the sum of the parameter scores classifies the sections as natural, altered and impacted. From the selected sections, only one in each basin was natural, the others altered or impacted. The elaborated protocol was validated by the application of the Biological Monitoring Working Party (BMWP) index which uses benthic macroinvertebrates as bioindicators. This validation demonstrated similar results in $50 \%$ of the evaluated sections, with a positive correlation between RAP and high BMWP $(r=0,85)$. Thus, the use of RAP can be considered an efficient assessment tool, in addition to providing participatory and integrated management that is recommended by the National Water Resources Policy.

Keywords: Habitat Assessment. Water Resources. Environmental Management.

\section{Introdução}

Os rios são considerados ecossistemas naturalmente hierárquicos e heterogêneos em múltiplas escalas espaciais (FRISSELL et al., 1986), que interagem permanentemente com as bacias hidrográficas em que estão inseridos (TUNDISI; TUNDISI, 2008). Entre as atividades que causam impacto nestas paisagens, está a intensa exploração de áreas naturais para produção agrícola e pecuária (COELHO et al., 2014), urbanização e industrialização (BENTOS et al., 2018). As atividades antrópicas alteram as paisagens, principalmente quando estão inseridas em ambientes de zonas ripárias, por meio da conversão de grandes áreas naturais para agricultura e pastagem. Estas mudanças na escala da bacia hidrográfica incluem fatores como o desmatamento das áreas ribeirinhas, que impactam os sistemas de água por meio da sedimentação e degradação da qualidade da água (química, física e características biológicas) e perda de biodiversidade (ALLAN, 2004; SAITO et al., 2016).

Uma das formas de avaliação da qualidade das águas nos riachos é integrar fatores ecológicos e fatores de qualidade do meio físico (SARAIVA, 1999; RODRIGUES; CASTRO, 2008a). Isto porque os ecossistemas aquáticos são integrados por componentes e processos bem mais amplos do que apenas o componente água (RODRIGUES; CASTRO, 2008b). Fatores topográficos como estabilidade das margens, sinuosidade do leito e uso e ocupação da terra no entorno dos riachos podem contribuir para uma maior ou menor entrada de material alóctone nos corpos hídricos, afetando as comunidades que deles dependem (HEPP et al., 2010; SENSOLO et al., 2012). Assim, é crescente a necessidade de avaliar e monitorar as alterações 
ambientais e seus efeitos sobre os recursos hídricos, principalmente no que se refere ao desenvolvimento de metodologias usadas como instrumentos para medir a "saúde" dos ecossistemas aquáticos (RODRIGUES; CASTRO, 2008a; CALLISTO et al., 2019).

Por volta de 1980, os órgãos ambientais passaram a definir métodos de avaliação qualitativos. A Enviromental Protection Agency (EPA, 1987), juntamente com agências de monitoramento das águas superficiais nos Estados Unidos, deu início, em 1986, a estudos com relação à qualidade da água e apresentou seus resultados em 1987, os quais deram origem ao relatório "Surface Water Monitoring: A Framework for Change" (RODRIGUES; CASTRO, 2008b). Este relatório apresentava a reestruturação dos programas de monitoramento e o auxílio em pesquisas, possibilitando o início do desenvolvimento de protocolos de análise rápida com baixo custo.

Desta forma, surgem os Protocolos de Avaliação Rápida (PAR) largamente utilizados em países como Estados Unidos, Austrália e Reino Unido (PLAFKIN et al., 1989; HANNAFORD et al., 1997; BARBOUR et al., 1999). Esses primeiros protocolos serviram como base para o surgimento de diversos modelos, os quais são instrumentos que levam em consideração a análise integrada dos ecossistemas lóticos, e seu entorno, por meio de uma metodologia fácil, simples e viável para a aplicação pelas mais diferentes pessoas (CALLISTO et al., 2002). No Brasil, diversos estudos utilizando PAR analisaram os ecossistemas aquáticos de forma estrutural e funcional, entre eles: Callisto et al. (2002) para Minas Gerais e Rio de Janeiro, Rodrigues, Castro (2008b) também para Minas Gerais; Lobo et al. (2012) na sub-bacia do Rio Pardinho, no Rio Grande do Sul; Cajaiba et al. (2015) na Paraíba, Rigotti et al. (2016) para Santa Catarina, na região da grande Florianópolis; Brito et al. (2016) para o semiárido brasileiro.

Os PAR podem ser um componente importante em Programas de Monitoramento Ambiental, oferecendo oportunidade para avaliar os níveis de impactos antrópicos em trechos de bacias hidrográficas, ou contribuindo para a conservação de recursos naturais (CALLISTO et al., 2002; RODRIGUES; CASTRO, 2008c; FRANÇA; SALLES, 2010; BENTOS et al., 2018). O uso dos PAR favorece a participação social, pois auxilia na construção de estruturas de relacionamento entre estado e sociedade, buscando melhorias a interesses da coletividade, sendo fundamental na consolidação da democracia (CARVALHO, 1998; NASCIMENTO, 1997; EVERSOLE, 2003), bem como para políticas e programas de gerenciamento de recursos hídricos (MMA, 2016). Os PAR são ferramentas metodológicas de rápida aplicação e fácil compreensão, utilizadas para avaliar os níveis de conservação das condições ambientais em trechos de rios (BENTOS et al., 2018).

Estes protocolos foram criados visando à necessidade de monitoramento contínuo dos riachos, a fim de reduzir o dano ambiental ocasionado aos mesmos. Porém, em virtude da grande diversidade de ecossistemas existentes no Brasil, os protocolos acabam necessitando de adaptações para melhor avaliarem a "saúde" dos recursos hídricos em nível local, já que existem grandes variações na estrutura desses ambientes (CALLISTO et al., 2002; FIRMINO et al., 2011).

Desta forma, considerando características dos riachos do Sul do Brasil, o objetivo deste estudo foi elaborar um Protocolo de Avaliação Rápida para trechos de riachos de cabeceira em Mata Atlântica no Sul do Brasil, visando à avaliação da diversidade de habitats e do nível de preservação, como ferramenta para a gestão de bacias hidrográficas. 


\section{Material e Métodos}

\section{Área de Estudo}

Para a elaboração do protocolo, inicialmente selecionou-se uma bacia hidrográfica no estado de Santa Catarina, neste caso do rio Tamanduá $\left(27^{\circ} 17^{\prime} 21^{\prime \prime}\right.$ a $27^{\circ} 20^{\prime} 17^{\prime \prime} \mathrm{S}$ e $51^{\circ} 58^{\prime} 41^{\prime \prime}$ a $\left.52^{\circ} 02^{\prime} 59^{\prime \prime} \mathrm{O}\right)$, tendo suas nascentes localizadas em áreas rurais e urbanizadas do município de Concórdia, SC; e uma no estado do Rio Grande do Sul, rio Dourado $\left(27^{\circ} 35^{\prime} 25^{\prime \prime}\right.$ a $27^{\circ} 38^{\prime} 21^{\prime \prime}$ S e $52^{\circ} 15^{\prime} 21^{\prime \prime}$ a $52^{\circ} 18^{\prime} 59^{\prime \prime}$ ), com nascentes na área urbana e rural do município de Erechim, RS (Figura 1). Ambas as bacias hidrográficas são pertencentes ao bioma Mata Atlântica.
A escolha dessas duas bacias ocorreu em função de serem semelhantes quanto a características de usos e ocupação da terra e de características morfométricas (Tabela I, Figuras $2 \mathrm{a}$ e $2 \mathrm{~b}$ ). As características morfométricas das áreas de drenagem se referem a topografia (clinografia) e geomorfologia de duas Bacias Hidrográficas em estudo. Para tanto, utilizou-se de dados cartográficos já existentes no Laboratório de Geoprocessamento e Planejamento Ambiental (LagePlam) da URI Erechim. Os parâmetros avaliados para cada bacia hidrográfica (Rio Tamanduá e Rio Dourado) seguiram a metodologia apresentada por CHRISTOFOLETTI (2007).

Quanto aos usos e ocupação da terra, a bacia do Rio Dourado apresenta um predomí-

Figura 1 - Localização das Bacias Hidrográficas - Rio Tamanduá (Concórdia/SC) e Rio Dourado (Erechim/RS)

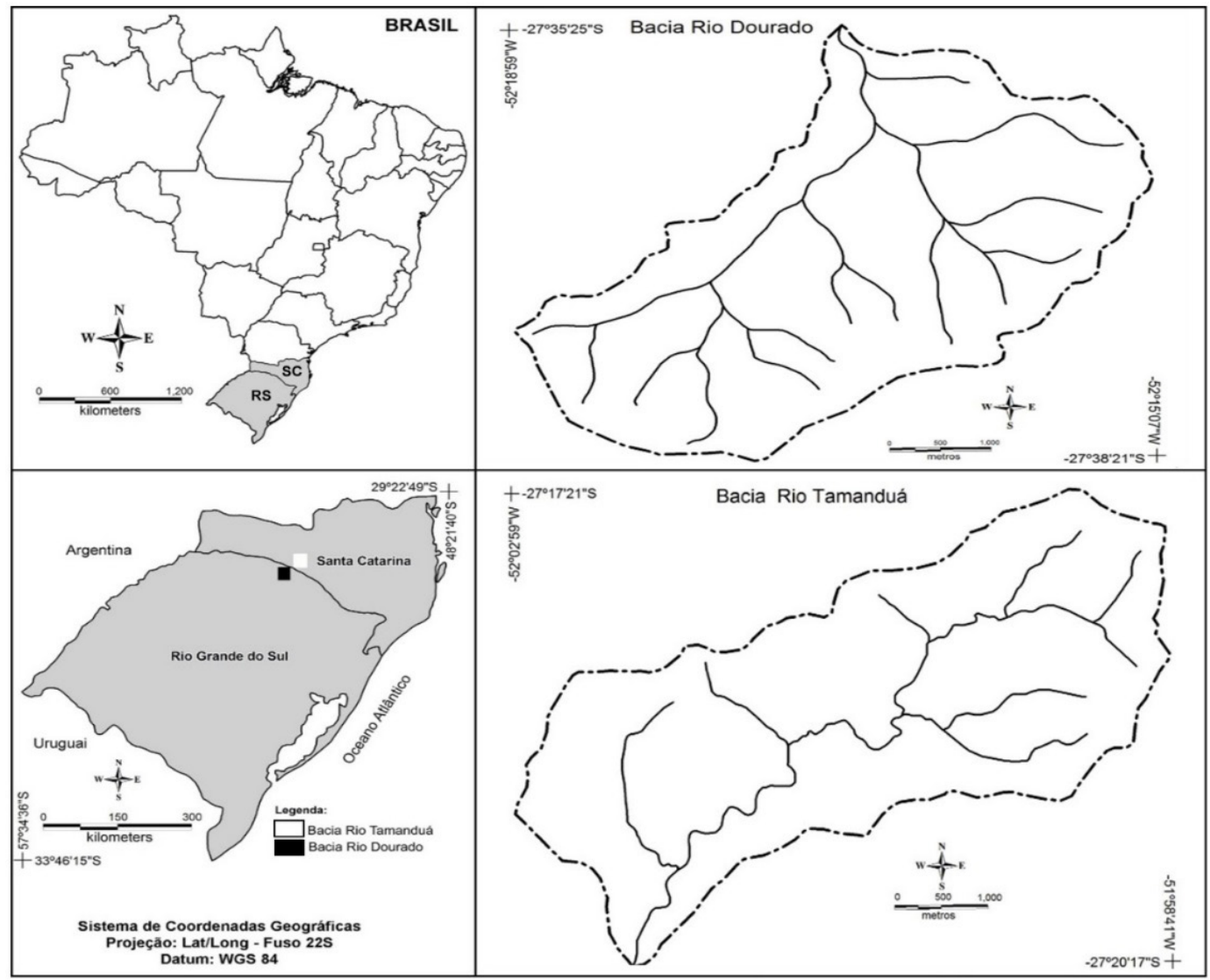


nio de cobertura por vegetação arbórea nativa $(58,7 \%)$, apresentando área urbanizada em $10,8 \%$ da área da bacia. A silvicultura perfaz $9,27 \%$. A bacia do Rio Tamanduá também apresenta maior percentagem de vegetação arbórea nativa (48,06\%), seguida de silvicultura com um total de $12,38 \%$ da área. Essas duas bacias serviram de modelo para coleta de informações e elaboração do protocolo.

Figura 2 - Uso e cobertura da terra nas Bacias Hidrográficas: a) Rio Dourado/RS; b) Rio Tamanduá/SC

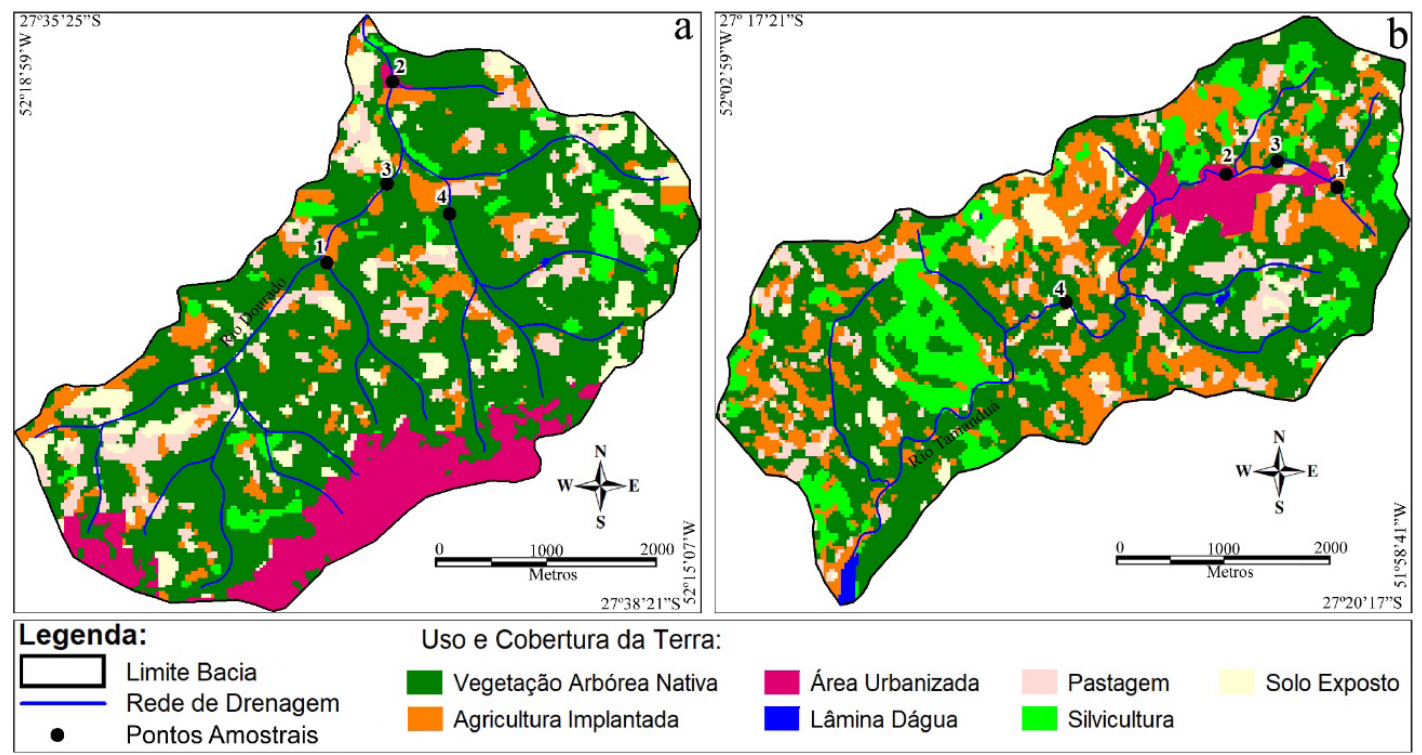

Tabela I - Parâmetros morfométricos das áreas de drenagem das Bacias Hidrográficas Rio Dourado/RS e Rio Tamanduá/SC (maio/2016)

\begin{tabular}{lcc}
\hline \multicolumn{1}{c}{ Item } & Rio Dourado & Rio Tamanduá \\
\hline Área (ha) & 1.807 & 1.873 \\
Perímetro (km) & 19,02 & 20,71 \\
Valor Somatório do comprimento das Curvas de Nível $(\mathrm{km})$ & 256,5 & 220,0 \\
Equidistância (m) & 20 & 20 \\
Comprimento do Rio Principal (km) & 6,378 & 9,853 \\
Altitude da Nascente do Rio Principal (m) & 755 & 600 \\
Altitude da Foz (m) & 545 & 355 \\
Somatório de Rios (km) & 25,52 & 20,69 \\
Declividade Média (\%) & 28,39 & 23,49 \\
Densidade de Drenagem (m/ha) & 14,122 & 11,04 \\
Índice de Circularidade da bacia & 0,627 & 0,5487 \\
Coeficiente de Rugosidade $\left(\mathrm{km} / \mathrm{km}^{2}\right)$ & 40,09 & 25,93 \\
Gradiente do Canal Principal & 32,92 & 24,86 \\
\hline
\end{tabular}




\section{Elaboração do Protocolo}

Para a elaboração do PAR, após a escolha das duas bacias hidrográficas, foram estabelecidas algumas etapas: definição dos riachos, levantamento de informações ambientais dos locais, elaboração do Protocolo, aplicação, validação e consolidação do Protocolo (Figura 3). Quatro trechos foram selecionados nas duas bacias hidrográficas, baseando-se nos usos e ocupação da terra e características morfométricas. Em cada trecho realizou-se um levantamento in loco de parâmetros físicos, como tipo de substrato, presença de vegetação, de agricultura, de área urbanizada, entre outros, para compor o protocolo. Com os parâmetros definidos, procedeu-se a caracterização dos mesmos e a possível pontuação para cada um deles, utilizando-se como base os protocolos de Barbour et al. (1999), Callisto et al. (2002) e Rodrigues; Castro (2008c). Para os parâmetros relacionados a vegetação foi definido, como raio de observação, $30 \mathrm{~m}$, baseado no Código Florestal, Lei 12.651/2012 (BRASIL, 2012).

Foram elencados 19 parâmetros com respectivas características (Quadro 1). Para cada característica do parâmetro atribui-se uma pontuação que varia entre 0 (zero), 2 ou 4 pontos. Os valores são distribuídos de acordo com uma condição de estresse ambiental. O somatório da pontuação dos parâmetros pode variar indicando se a condição do local analisado é natural, alterado ou impactado (Tabela II).

Com os trechos e parâmetros selecionados, elaborou-se a primeira versão do protocolo, que foi aplicado na Bacia Hidrográfica do rio Dourado/RS e na Bacia do rio Tamanduá/SC, por alunos do laboratório de Biomonitoramento da URI. Após esta aplicação, verificou-se a necessidade de adequação, pois algumas características geravam dúvidas entre os avaliadores, e os resultados obtidos não condiziam com a situação visualizada. Visando a auxiliar na aplicação do PAR, foi elaborado um material de apoio para auxiliar na aplicação do Protocolo. Esse material constou de uma cartilha contendo definição de termos ecológicos e explicações sobre caraterísticas utilizadas em cada parâmetro.

Figura 3 - Fluxograma referente às etapas para a elaboração do Protocolo

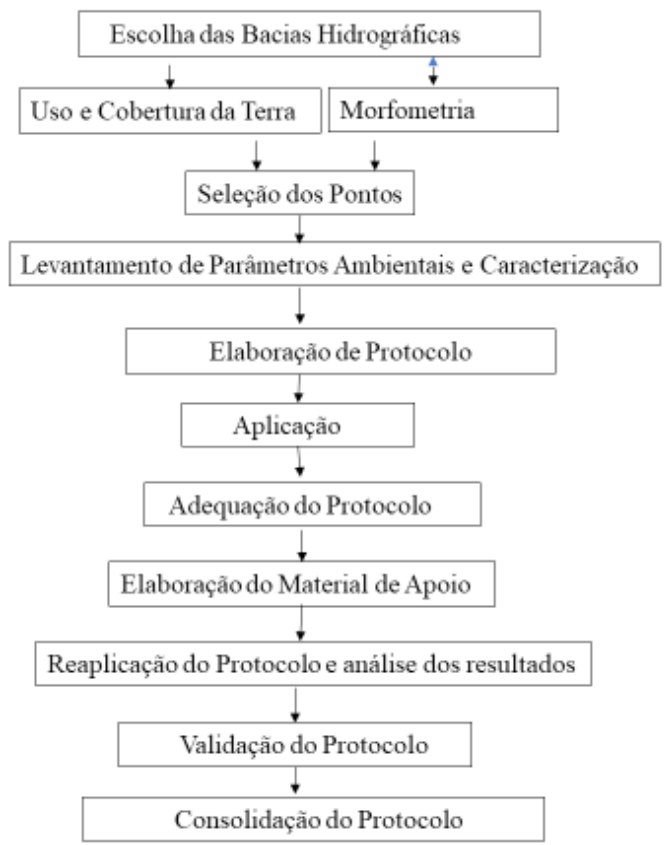

Posteriormente, foi selecionado um grupo de 8 voluntários, com idade entre 18 e 25 anos, em cada uma das bacias hidrográficas. Alguns possuíam o ensino médio incompleto e outros, finalizando o curso de Ciências Biológicas. Quatro voluntários utilizaram o material de apoio e quatro realizaram a aplicação do PAR sem o material de apoio. De posse dos resultados obtidos nas duas bacias hidrográficas, passou-se à validação do protocolo. 
Tabela II - Pontuação final do PAR correspondente a condições ecológicas dos trechos de riachos de cabeceira em Mata Atlântica, Sul do Brasil

\begin{tabular}{cc}
\hline Condições ecológicas do trecho & Pontuação \\
\hline Natural & Mais de 65 \\
Alterado & 40 a 64 \\
Impactado & 0 a 39 \\
\hline
\end{tabular}

Quadro 1 - Protocolo de avaliação rápida para trechos de riachos de cabeceira em Mata Atlântica, Sul do Brasil

\begin{tabular}{|c|c|c|c|}
\hline \multicolumn{4}{|l|}{ Município/Estado: } \\
\hline \multicolumn{4}{|l|}{ Coordenadas geográficas: } \\
\hline \multicolumn{4}{|l|}{ Data da avaliação: } \\
\hline Parâmetro & 4 pontos & 2 pontos & 0 pontos \\
\hline Odor da água & Nenhum & $\begin{array}{l}\text { Causas naturais (algas, } \\
\text { vegetais em decompo- } \\
\text { sição) }\end{array}$ & $\begin{array}{l}\text { Causas artificiais (esgoto } \\
\text { doméstico e industrial) }\end{array}$ \\
\hline Transparência da água & Transparente & Turva/cor de chá forte & Opaca ou colorida \\
\hline Agricultura & Inexistente & $\begin{array}{l}\text { Existente a um raio maior } \\
\text { que } 30 \mathrm{~m}\end{array}$ & $\begin{array}{c}\text { Existente a um raio me- } \\
\text { nor que } 30 \mathrm{~m}\end{array}$ \\
\hline Área industrial & Inexistente & $\begin{array}{c}\text { Existente a um raio maior } \\
\text { que } 30 \mathrm{~m}\end{array}$ & $\begin{array}{l}\text { Existente a um raio me- } \\
\text { nor que } 30 \text { metros }\end{array}$ \\
\hline Área urbanizada & Inexistente & $\begin{array}{c}\text { Existente a um raio maior } \\
\text { que } 30 \mathrm{~m}\end{array}$ & $\begin{array}{c}\text { Existente a um raio me- } \\
\text { nor que } 30 \mathrm{~m}\end{array}$ \\
\hline $\begin{array}{c}\text { Uso/alteração do solo do } \\
\text { entorno }\end{array}$ & $\begin{array}{l}\text { Não apresentando presença } \\
\text { de estrada em um raio de } 30 \\
\text { m, ou qualquer outro tipo de } \\
\text { alteração/obra no trecho }\end{array}$ & $\begin{array}{c}\text { Estradas a menos de } 30 \mathrm{~m} \\
\text { do riacho com movimen- } \\
\text { tação de solo em uma das } \\
\text { margens }\end{array}$ & $\begin{array}{l}\text { Estradas a menos de } 30 \mathrm{~m} \\
\text { do riacho com movimen- } \\
\text { tação de solo nas duas } \\
\text { margens }\end{array}$ \\
\hline $\begin{array}{l}\text { Criação de gado e/ou } \\
\text { suíno e/ou frango }\end{array}$ & Inexistente & $\begin{array}{c}\text { Existente a um raio maior } \\
\text { que } 30 \mathrm{~m}\end{array}$ & $\begin{array}{c}\text { Existente a um raio me- } \\
\text { nor que } 30 \mathrm{~m}\end{array}$ \\
\hline Sinuosidade do leito & $\begin{array}{c}\text { Presença de curvas evidentes } \\
\text { proporcionando diversidade } \\
\text { de habitats }\end{array}$ & $\begin{array}{c}\text { A sinuosidade do canal é } \\
\text { pouco evidente, podendo } \\
\text { ser observadas curvas } \\
\text { distantes }\end{array}$ & $\begin{array}{l}\text { O trecho apresenta-se } \\
\text { retilíneo }\end{array}$ \\
\hline Estabilidade das margens & $\begin{array}{c}\text { Estáveis, ausência ou míni- } \\
\text { ma evidência de erosão, com } \\
\text { menos de } 5 \% \text { das margens } \\
\text { afetada }\end{array}$ & $\begin{array}{l}\text { Estabilidade comprome- } \\
\text { tida, apresentando de } 5 \\
\text { a } 50 \% \text { das margens com } \\
\text { probabilidade de erosão }\end{array}$ & $\begin{array}{l}\text { Instáveis, apresentando } \\
\text { mais de } 50 \% \text { das margens } \\
\text { com presença de erosão } \\
\text { ou quedas de blocos de } \\
\text { solo ou rocha }\end{array}$ \\
\hline
\end{tabular}




\begin{tabular}{|c|c|c|c|}
\hline Alteração no canal do rio & $\begin{array}{l}\text { Ausência de canalização, ou } \\
\text { qualquer outra perturbação; } \\
\text { o curso d'água segue o pa- } \\
\text { drão natural }\end{array}$ & Presença de ponte & $\begin{array}{c}\text { Presença de canalização, } \\
\text { "passador" ou outras } \\
\text { formas de alteração } \\
\text { (terraplanagem, aterros, } \\
\text { barragens, margens re- } \\
\text { vestidas) }\end{array}$ \\
\hline $\begin{array}{c}\text { Erosão no entorno do } \\
\text { ponto e/ou nas margens } \\
\text { do rio }\end{array}$ & Ausente & Moderada & Acentuada \\
\hline Corredeiras & $\begin{array}{l}\text { Corredeiras frequentes; entre } \\
\text { as corredeiras há a formação } \\
\text { de pequenos remansos, com } \\
\text { aumento na quantidade de } \\
\text { habitats }\end{array}$ & $\begin{array}{l}\text { Corredeiras frequentes } \\
\text { e não há condições para } \\
\text { a presença de habitats } \\
\text { favoráveis }\end{array}$ & $\begin{array}{c}\text { Inexistência de corre- } \\
\text { deiras; a maior parte da } \\
\text { superfície da água é plana } \\
\text { ou encontra-se parada em } \\
\text { poços }\end{array}$ \\
\hline Tipo de habitat de fundo & $\begin{array}{l}\text { Mais de } 50 \% \text { com presença } \\
\text { de pedras, cascalho, folhas, } \\
\text { troncos e galhos; observa-se } \\
\text { também seixos, ou outros ha- } \\
\text { bitats disponíveis para a co- } \\
\text { lonização e abrigo de insetos } \\
\text { aquáticos, anfíbios ou peixes }\end{array}$ & $\begin{array}{l}\text { Entre } 20 \text { e } 50 \% \text { com pre- } \\
\text { sença de pedras, cascalho, } \\
\text { folhas, troncos; há subs- } \\
\text { tratos adicionais (troncos } \\
\text { ou galhos) inclinados, } \\
\text { mas que ainda não fazem } \\
\text { parte do fundo }\end{array}$ & $\begin{array}{l}\text { Menos de } 20 \% \text { com pre- } \\
\text { sença de pedras, cascalho, } \\
\text { folhas, troncos; pouca di- } \\
\text { versidade de habitats }\end{array}$ \\
\hline $\begin{array}{l}\text { Deposição de lama (sedi- } \\
\text { mentos finos) }\end{array}$ & $\begin{array}{l}\text { A deposição de lama/sedi- } \\
\text { mento afeta menos de } 20 \% \\
\text { do substrato do riacho }\end{array}$ & $\begin{array}{c}\text { A deposição de lama/sedi- } \\
\text { mento afeta de } 20 \text { a } 50 \% \\
\text { do substrato do riacho }\end{array}$ & $\begin{array}{l}\text { A deposição de lama/ } \\
\text { sedimento afeta mais } \\
\text { de } 50 \% \text { do substrato do } \\
\text { riacho }\end{array}$ \\
\hline $\begin{array}{c}\text { Presença de serapilheira } \\
\text { nas margens }\end{array}$ & Abundante & Pouco abundante & Inexistente \\
\hline $\begin{array}{c}\text { Vegetação nativa em um } \\
\text { raio de } 30 \mathrm{~m}\end{array}$ & $\begin{array}{c}\text { Totalizando mais de } 60 \% \text { em } \\
\text { ambas as margens }\end{array}$ & $\begin{array}{c}\text { Entre } 20 \text { e } 60 \% \text { em ambas } \\
\text { as margens }\end{array}$ & $\begin{array}{c}\text { Menos de } 20 \% \text { em ambas } \\
\text { as margens }\end{array}$ \\
\hline $\begin{array}{c}\text { Vegetação exótica em um } \\
\text { raio de } 30 \mathrm{~m}\end{array}$ & Ausente & $\begin{array}{c}\text { Compondo até } 30 \% \text { da } \\
\text { área }\end{array}$ & $\begin{array}{c}\text { Compondo mais de } 30 \% \\
\text { da área }\end{array}$ \\
\hline
\end{tabular}

Atenção: Para o quesito de cobertura de dossel, leve em consideração as características da vegetação nativa de sua região, utilizando apenas um dos dois próximos parâmetros, sendo o $1^{\circ}$ para áreas onde a vegetação nativa apresenta características arbóreas e o $2^{\circ}$ para áreas de característica de campo natural.

\begin{tabular}{|c|c|c|c|}
\hline $\begin{array}{c}\text { Dossel (áreas com vegeta- } \\
\text { ção nativa arbórea) }\end{array}$ & $\begin{array}{c}\text { Dossel fechado, apresentan- } \\
\text { do cobertura superior a 60\% } \\
\text { do rio }\end{array}$ & $\begin{array}{c}\text { Dossel apresenta cobertu- } \\
\text { ra entre 30 e 60\% parcial } \\
\text { do rio }\end{array}$ & $\begin{array}{c}\text { Dossel aberto, cobertura } \\
\text { inferior a 30\% }\end{array}$ \\
\hline $\begin{array}{c}\text { Dossel (com vegetação } \\
\text { nativa de campo natural) }\end{array}$ & Dossel Aberto & & \\
\hline
\end{tabular}

\section{Total de pontos:}




\section{Validação do Protocolo Elaborado}

O PAR foi aplicado em mais 10 riachos da região do município de Erechim, RS, para validação das informações. A validação foi realizada a partir da correlação dos resultados obtidos pelo PAR e pelas informações biológicas disponíveis na base de dados do Laboratório de Biomonitoramento da URI (para mais detalhes sobre metodologia de coleta e identificação, ver (HEPP; RESTELLO, 2007). As informações biológicas foram analisadas utilizando-se o índice Biological Monitoring Working Party (BMWP), adaptado de JUNQUEIRA et al. (2000). O BMWP baseia-se no somatório de valores de tolerância (sensibilidade a poluentes orgânicos) atribuídos a cada família de macroinvertebrados, de acordo com sua capacidade de sobreviver em diferentes situações de qualidade de água (JUNQUEIRA; CAMPOS, 1998; BISPO, 2006). Para análise utilizou-se correlação de Pearson com o auxílio do software "R" (R CORE TEAM, 2018).

\section{RESULTADOS E DISCUSSÃO}

\section{Aplicação do Protocolo por Voluntários}

Os resultados da aplicação pelos voluntários com e sem material de apoio podem ser visualizados na Tabela III. Verifica-se que os resultados são homogêneos e a diferença entre avaliadores não ultrapassa 4 pontos. $\mathrm{O}$ tempo médio de preenchimento do PAR para os voluntários com material de apoio foi de 18 a 20 minutos. Para os voluntários sem material de apoio o tempo de aplicação diminuiu para 12 minutos. A utilização do material de apoio não interferiu nos resultados obtidos, porém seu uso auxiliou os usuários a diminuir o tempo de aplicação, demonstrando maior agilidade e compreensão dos parâmetros e características que estão sendo analisadas.

Muito embora já tenham sido propostos vários Protocolos de Avaliação Rápida para diferentes regiões no Brasil, como Callisto et al. (2002), Bersot et al. (2015), Rigotti et al. (2016), Brito et al. (2016), estes ainda são pouco difundidos e utilizados, não atingindo todo o potencial de monitoramento que possuem. Os protocolos de análise rápida podem ser alterados conforme os diferentes tipos de vegetação, clima, solo, altitude, ou mesmo conforme diferentes países (BIZZO et al., 2014). O PAR é um método de análise com baixo custo, de fácil aplicação e obtenção de respostas rápidas (CALLISTO et al., 2001; RODRIGUES; CASTRO, 2008b), fato observado quando da aplicação desta proposta.

O protocolo elaborado apresenta linguagem clara e de fácil interpretação dos resultados. A utilização desta ferramenta permite que toda a sociedade possa participar de

Tabela III - Pontuação obtida pelos avaliadores utilizando-se o Protocolo de Avaliação Rápida com e sem material de apoio (riachos de cabeceira, Mata Atlântica, Sul do Brasil)

\begin{tabular}{ccccc|ccccc}
\hline \multicolumn{3}{c}{ Sem material de apoio } & \multicolumn{7}{c}{ Com material de apoio } \\
\hline Avaliador & R1 & $\mathbf{R 2}$ & $\mathbf{R 3}$ & $\mathbf{R 4}$ & Avaliador & $\mathbf{R 1}$ & $\mathbf{R 2}$ & $\mathbf{R 3}$ & $\mathbf{R 4}$ \\
\hline $\mathbf{1}$ & 40 & 62 & 22 & 66 & $\mathbf{1}$ & 42 & 64 & 18 & 64 \\
$\mathbf{2}$ & 42 & 64 & 18 & 64 & $\mathbf{2}$ & 44 & 66 & 20 & 62 \\
$\mathbf{3}$ & 44 & 64 & 20 & 62 & $\mathbf{3}$ & 44 & 66 & 20 & 64 \\
$\mathbf{4}$ & 40 & 66 & 18 & 64 & $\mathbf{4}$ & 42 & 68 & 20 & 64 \\
$\mathbf{5}$ & 42 & 64 & 18 & 66 & $\mathbf{5}$ & 46 & 68 & 18 & 60 \\
$\mathbf{6}$ & 40 & 62 & 20 & 64 & $\mathbf{6}$ & 42 & 66 & 22 & 64 \\
$\mathbf{7}$ & 40 & 64 & 20 & 66 & $\mathbf{7}$ & 42 & 68 & 20 & 60 \\
$\mathbf{8}$ & 40 & 62 & 22 & 62 & $\mathbf{8}$ & 44 & 62 & 18 & 62 \\
\hline
\end{tabular}


processos de monitoramento e gerenciamento dos recursos hídricos (...). O PAR elaborado demostrou ser eficaz por permitir a apropriação de conceitos ecológicos, a sensibilização e observação da interação dos recursos hídricos com seu entorno, o que permite ser utilizado por pesquisadores, pós-graduandos, em escolas, pela população em geral, ONGs e também auxiliar nas atividades de Educação Ambiental e inclusive visando a recuperação de rios (CALLISTO et al., 2022; GUIMARÃES et al., 2012; HABBERFIELD et al., 2014). Cabe salientar que os PARs devem ser adaptados às condições regionais devido a características próprias que integram os ambientes em níveis regionais.

Comparando este protocolo com o de Hannaford et al. (1997), verifica-se que cada parâmetro de avaliação recebia de 0 a 20 pontos, o que certamente contribui para a variação entre as respostas (CALLISTO et al., 2002). Por outro lado, o protocolo de Callisto et al. (2002) acabou simplificando a pontuação para no máximo 5 pontos, de modo a favorecer a aplicação. No protocolo elaborado, o máximo para cada parâmetro avaliado é 4 pontos. Isto favorece não só a alunos de graduação, pós-graduação, de escolas, bem como a pessoas sem formação na área, tendo em vista a popularização da informação científica.

\section{Avaliação Ambiental dos Trechos Selecionados}

$\mathrm{Na}$ bacia do Rio Dourado, o protocolo aplicado indicou o trecho 4 como sendo natural (Tabela IV). Este possui características ecológicas essenciais, como vegetação arbórea nativa em um raio de $30 \mathrm{~m}$, com diferentes espécies; presença da serapilheira e margens estáveis. Enquanto o trecho 2, considerado impactado, apresenta modificações como canalização, depósito de lama, sedimento fino, menor heterogeneidade e menos de $20 \%$ de vegetação ripária (Figura $2 a$ ).
Na bacia do Rio Tamanduá, os trechos 1 e 4 foram considerados alterados (44 e 62 pontos, respectivamente) (Tabela IV). O trecho 2 foi considerado natural (68 pontos), e o 3 , com 20 pontos, impactado. O trecho considerado natural se caracteriza por apresentar vegetação arbórea nativa em um raio de $30 \mathrm{~m}$, com diferentes espécies, com serapilheira, tendo o substrato de fundo composto por pedras, cascalho, folhas e galhos, o que causa uma maior heterogeneidade no local. $\mathrm{O}$ trecho impactado (3), caracteriza-se por seu entorno estar basicamente ocupado por usos antrópicos (agricultura implantada, pastagem e solo exposto).

\section{Validação do Protocolo}

Como citado, o processo de validação do protocolo ocorreu por meio da aplicação do BMWP em comparação com o PAR (Tabela V). Observa-se, desta forma, que em $50 \%$ dos riachos os resultados foram semelhantes. Apenas os riachos 1, 4 e 7 apresentaram resultados diferentes entre os valores do protocolo e o índice BMWP. Verificou-se que estes riachos sofreram alterações como margens instáveis e processos erosivos, e os dados biológicos utilizados foram de anos anteriores. Pelo Coeficiente de Correlação de Pearson, verificou-se correlação positiva $(r=0,85)$ entre os resultados do BMWP e os resultados do Protocolo elaborado.

Em trabalho realizado por RIGOTTI et al. (2016) utilizando macroinvertebrados bentônicos, métodos analíticos de análise de água e protocolo de avaliação de habitats, comenta-se que testes de correlação cujo valor de r é igual ou superior a 0,80 os resultados indicam a efetividade dos protocolos na avaliação de trechos de rios, vindo a corroborar com os resultados aqui apresentados para os riachos de cabeceira em Mata Atlântica no Sul do Brasil.

A aplicação do protocolo elaborado em outros riachos, que não os selecionados inicialmente, foram importantes tendo em 
PROTOCOLO DE ANÁLISE RÁPIDA: ALTERNATIVA PARA AVALIAR QUALIDADE AMBIENTAL EM RIACHOS DE CABECEIRA EM MATA ATLÂNTICA, SUL DO BRASIL

Tabela IV - Resultados da aplicação do Protocolo de Avaliação Rápida para riachos de cabeira de Mata Atlântica

\begin{tabular}{ccc}
\hline Pontos & $\begin{array}{c}\text { Bacia do Rio Dourado/RS } \\
\text { (Pontuação/Avaliação) }\end{array}$ & $\begin{array}{c}\text { Bacia do Rio Tamanduá/SC } \\
\text { (Pontuação/Avaliação) }\end{array}$ \\
\hline 1 & $60 /$ alterado & $44 /$ alterado \\
2 & $18 /$ impactado & $68 /$ natural \\
3 & $48 /$ alterado & $20 /$ impactado \\
4 & $68 /$ natural & $62 /$ alterado \\
\hline
\end{tabular}

Tabela V - Resultado obtido pelo índice BMWP e pela aplicação do Protocolo de Avaliação Rápida em riachos do município de Erechim, RS

\begin{tabular}{ccccc}
\hline Riachos & $\begin{array}{c}\text { Protocolo } \\
\text { Elaborado }\end{array}$ & Categoria Protocolo & BMWP & Categoria BMWP \\
\hline R1 & 60 & Alterado & 90 & Excelente \\
R 2 & 50 & Alterado & 60 & Regular \\
R 3 & 38 & Impactado & 46 & Regular \\
R 4 & 46 & Alterado & 70 & Boa \\
R 5 & 72 & Natural & 99 & Excelente \\
R 6 & 18 & Impactado & 2 & Péssima \\
R 7 & 38 & Impactado & 74 & Boa \\
R 8 & 22 & Impactado & 58 & Regular \\
R 9 & 66 & Natural & 86 & Excelente \\
R 10 & 32 & Impactado & 32 & Ruim \\
\hline
\end{tabular}

vista a sua utilização de forma sistemática em qualquer trecho de riachos de Mata Atlântica do Sul do Brasil.

O resultado obtido na análise de correlação demonstra que os parâmetros utilizados são adequados ao ambiente proposto, fornecendo dados confiáveis com relação à qualidade do ambiente avaliado. Assim, o protocolo traz complementariedade aos dados bióticos, sendo adequado para a avaliação rápida de riachos de pequena ordem.

\section{Conclusão}

Os resultados apresentados pela aplicação do protocolo indicam que muitos trechos dos riachos avaliados mostram alterações na integridade ambiental, uma vez que se encontram alterados ou impactados. Tal resultado deve-se aos impactos antrópicos decorrentes do uso e cobertura da terra, em especial pelas atividades agrícolas ou urbanas. Desta forma, o protocolo elaborado apresenta-se como alternativa de avaliação de diversidade de $h a$ bitats e do nível de preservação de riachos de cabeceira na Mata Atlântica. Assim, este Protocolo de Avaliação Rápida pode ser utilizado por órgãos e entidades governamentais, como entidades educacionais, centros de pesquisa e de monitoramento de qualidade de água, sociedade civil organizada em suas diversas modalidades, Comitês de Gerenciamento de Bacias e a sociedade como um todo.

Este tipo de diagnóstico com uso de PARs facilita a inserção de todos na gestão dos recursos hídricos, conforme preconizado pela Política Nacional de Recursos Hídricos, visando a uma gestão participativa e integrada. 


\section{AGRADECIMENTOS}

Agradecemos ao Conselho Nacional de Desenvolvimento Científico e Tecnológico (CNPq) pelo apoio financeiro concedido a Rozane M. Restello (Processo no 409685/2016-0). JMS recebeu uma bolsa do Programa de Bolsas Universitárias de Santa Catarina (UNIEDU). Cristiane Biasi recebeu bolsa do Programa PNPD/CAPES/PPG Ecologia. Luiz U. Hepp recebe apoio financeiro do CNPq (421632/20160) e bolsa produtividade CNPq (305203/2017-7).

\section{REFERÊNCIAS}

ALLAN, J. D. Landscapes and rivers capes: The influence of land use on stream ecosystems. Annual Review of Ecology Evolution and Systematics, v. 35, p. 257-284, 2004.

BARBOUR, M. T.; GERRISTSEN, J.; SNYDER, B. D.; STRIBLING, J. B. Rapid bioassessment protocols for use in streams and wadeable rivers: periphyton, benthic macroinvertebrates and fish. 2. ed. Washington: EPA 841-B-99-002, 339p. 1999.

BENTOS, A. B.; GALlO, A. S.; GUIMARÃES, N. F.; SOUZA M. D. DE; STOLF, R.; BORGES, M. T. M. R. Rapid Assessment of Habitat Diversity Along the Araras Stream, Brazil. Floresta e Ambiente, v. 25, n. 1, e20160024, 2018.

BERSOT, M.R.O.B.; MENEZES, J.M.; ANDRADRE, S.F. DE. Aplicação do Protocolo de Avaliação Rápida de Rios (PAR) na bacia hidrográfica do rio Imbé-RJ. Ambiência, v. 11, n. 2, p. 277-294, 2015.

BISPO, P. C. Distribuição espacial de insetos aquáticos (Ephemeroptera, Plecoptera e Trichoptera) em córregos de cerrado do Parque Ecológico de Goiânia, Estado de Goiás. In: NESSIMIAN, J. L.; CARVALHO, A. L. E. (Ed.). Ecologia de insetos aquáticos. Rio de Janeiro: PPGE-UFRJ, 1998. cap. 13. (Series Oecologia Brasiliensis, 5). 2006.

BIZZO, M.; MENEZES, J. M.; ANDRADE, S. F. Protocolo de Avaliação Rápida de Rios (PAR). Caderno de Estudos Geoambientais, v. 4, n.1, p.5-13, 2014.

BRASIL. Lei no 12.651 de 25 de maio de 2012, da Presidência da República. Diário Oficial da União. Seção 1. p. 1, 28 de maio de 2012.

BRITO, M. T. S.; NASCIMENTO FILHO, S. L.; VIANA, G. F. S.; MELO JÚNIOR, M. Aplicação de um protocolo de avaliação ambiental rápida em dois reservatórios do semiárido brasileiro.

Brazilian Journal Aquatic Sciense Technology, v. 20, n. 1, p. 1-5, 2016.

CAJAIBA, R. L.; SILVA, W. B. da.; FEITOSA, A. K.; BRUXEL, M. Avaliação ambiental de um curso d água no município de Uruará, PA, através de um Protocolo de Avaliação Rápida. Cadernos de Agroecologia, v. 10, n. 3, p. 1-5, 2015.

CALLISTO, M.; MORETTI, M.; GOULART, M. Macroinvertebrados bentônicos como ferramenta para avaliar a saúde de riachos. Revista Brasileira de Recursos Hídricos, v. 6, n. 1, p. 71-82, 2001.

CALLISTO, M.; FERREIRA, W. R.; MORENO, P.; GOULART, M.; PETRUCIO, M. Aplicação de um protocolo de avaliação rápida da diversidade de habitats em atividades de ensino e pesquisa (MG-RJ). Acta Limnologica Brasiliensia, v.14, n. 1, p. 91-98. 2002. 
CALLISTO, M.; SILVA, D.R.O.; CARVALHO, D.R.; MACEDO, D.R.; CASTRO, D.M.P.; POMPEU, P.S.; BECKER, B.; SANCHES B.; SANTOS G.B.; ALVES C.B.M. Índices Multimétricos para Avaliação de Integridade Biótica. In: CALLISTO, M.; MACEDO, D. R.; CASTRO, D.M.P. DE; ALVES, C.M. (orgs.). Bases Conceituais para Conservação e Manejo de Bacias Hidrográficas. Belo Horizonte: Companhia Energética de Minas Gerais (CEMIG), 2019. CARVALHO, M.C. Participação social no Brasil hoje. São Paulo: Instituto Polis, 1998. CHRISTOFOLETTI, A. Modelagem de sistemas ambientais. São Paulo: Edgard Blücher, 2007. COELHO, V. H. R.; MONTENEGRO, S. M. G. L.; ALMEIDA. C. N.; DE LIMA, E. R. V.; NETO, A. R.; DE MOURA, G. S. S. Dinâmica do uso e ocupação do solo em uma bacia hidrográfica do semiárido brasileiro. Revista Brasileira de Engenharia Agrícola e Ambiental, v.18, n.1, p.64-72, 2014. EPA. United States Environmental Protection Agency. Surface Water Monitoring: A Framework for Change. Office of Water, of Office Policy Planning and Evaluation. Washington, DC, 1987.

EVERSOLE, R. Managing the pitfalls of participatory development: some insight from Australia. World Development, v. 31, n.5, p. 781-795, 2003

FIRMINO, P. F.; MALAFAIA, G.; RODRIGUES, A. S. L. Diagnóstico da integridade ambiental de trechos de rios localizados no município de Ipameri, Sudeste do Estado de Goiás, através de um protocolo de avaliação rápida. Brazilian Journal of Aquatic Science and Technology, v. 15, n. 2, p. 1-12, 2011.

FRANÇA, J; SALES, S. Avaliação ecológica da qualidade das águas (parâmetros físicos e químicos) dos riachos no RVS Mata do Junco, Capela, SE. In: III Encontro de Recursos Hídricos em Sergipe. 2010. Anais [...]. Sergipe: 2010.

FRISSELL, C. A.; LISS, W. J.; WARREN, C. E., HURLEY, M. D. A hierarchical framework for stream habitat classification - viewing streams in a watershed context. Environmental Management, v. 10, p. 199-214, 1986.

GUIMARÃES, A.; RODRIGUES, A. S. L.; MALAFAIA, G. Adequação de um protocolo de avaliação rápida de rios para ser usado por estudantes do ensino fundamental. Revista Ambiente \& Água, v. 7, n. 3, p. 241-260, 2012.

HABBERFIELD, M. W.; BLERSCH, S. S.; BENNETT, S. J.; ATKINSON, J. F. Rapid geomorphic and habitat stream assessment techniques inform restoration differently based on levels of stream disturbance. Journal of the American Water Resources Association, v. 50, n. 4, p. 1051-1062, 2014. HANNAFORD, M. J.; BARBOUR, M. T.; RESH, V. H. Training reduces observer variability in visual-based assessments of stream habitat. Journal of the North American Benthological Society, v. 16, n. 4, p. 853-860, 1997.

HEPP, L. U.; RESTELLO, R. M. Macroinvertebrados bentônicos como bioindicadores da qualidade das águas do Alto Uruguai Gaúcho. In: ZAKRZEVSKI, S. B. (Org.) Conservação e uso sustentável da água: múltiplos olhares. Erechim, RS: EdiFAPES, p. 75-86, 2007.

HEPP, L. U.; MILESI, S. V.; BIASI, C.; RESTELLO, R. M. Effects of agricultural and urban impacts on macroinvertebrates assemblages in streams (Rio Grande do Sul, Brazil). Zoologia, v. 27, n. 1, p. 106-113, 2010.

JUNQUEIRA, V. M.; CAMPOS, S. C. M. Adaptation of the "BMWP" method for water quality evaluation to Rio das Velhas watershed (Minas Gerais, Brazil). Acta Limnologica Brasiliensia, v. 10, n. 2, p.125-135, 1998.

LOBO, E.A.; VOOS, J.G.; ABREU JUNIOR, E.F. DE. Utilização de um protocolo de avaliação rápida de impacto ambiental em sistemas lóticos do Sul do Brasil. Caderno de Pesquisa, Série Biologia, v. 23, n. 1, p. 18-32, 2011. 
NASCIMENTO, E.P. Estado e Sociedade no Brasil: novos padrões de relacionamento? Brasília: Conselho de Justiça Federal, 1997.

PLAFKIN, J. L.; BARBOUR, M. T.; PORTER, K. D.; GROSS, S. K.; HUGHES, R. M. Rapid bioassessment protocols for use in streams and rivers: Benthic macroinvertebrates and fish. U.S. Environmental Protection Agency, Office of Water Regulations and Standards, Washington, D.C. EPA 440-4-89-001. 1989.

MMA. Resolução $\mathbf{N}^{\mathbf{0}}$ 181, de 7 de dezembro de 2016. Aprova as Prioridades, Ações e Metas do Plano Nacional de Recursos Hídricos para 2016-2020. Disponível em:

http://www.cnrh.gov.br/plano-nacional-de-recursos-hidricos/1974-resolucao-n-181-de-07-dedezembro-de-2016. Acesso em: 12 mar. 2017.

R Core Team. R: A Language and Environment for Statistical Computing. R

Foundation for Statistical Computing. Vienna. 2018. https://www.R-project.org.

RIGOTTI, J. A.; POMPÊO, C. A.; FONSECA, A. L. D. Aplicação e análise comparativa de três protocolos de avaliação rápida para caracterização da paisagem fluvial. Ambiente \& Água, v. 11, n. 1, p. 85-97, 2016.

RODRIGUES, A. S. L.; CASTRO, P. T. A. Protocolos de avaliação rápida de rios e a inserção da sociedade no monitoramento dos recursos. Revista Ambiente e Água: An Interdisciplinary Journal of Applied Science, v. 3, n.3, p. 143-155, 2008a.

RODRIGUES, A. S. L.; CASTRO, P. T. A. Protocolos de avaliação rápida: instrumentos complementares no monitoramento dos recursos hídricos. Revista Brasileira de Recursos Hídricos, v. 13, n.1, p.161-170, 2008b.

RODRIGUES, A. S. L.; CASTRO, P. T. A. Adaptation of a rapid assessment protocol for rivers on rocky meadows. Acta Limnologica Brasiliensia, v. 20, n. 4, p. 291-303, 2008 c.

SAITO, N. S.; MOREIRA, M. A.; SANTOS, A. R.; EUGENIO, F. C.; FIGUEIREDO, A. C. Geotecnologia e ecologia da paisagem no monitoramento da fragmentação florestal. Floresta e Ambiente, v. 23, n 2, p. 201-210, 2016.

SARAIVA, M.G.A.N. O rio como paisagem. Gestão de corredores fluviais no quadro do ordenamento do território. Lisboa: Fundação Calouste Gulbenkian e Fundação para a Ciência e Tecnologia, 1999.

SENSOLO, D.; HEPP, L. U.; DECIAN, V. S.; RESTELLO, R. M. Influence of landscape on the assemblages of Chironomidae. Annalles of Limnologie, v. 48, p. 391-400, 2012.

TUNDISI, J. G.; TUNDISI, T. M. Limnologia. São Paulo: Oficina de Textos, 2008. 